\title{
An Efficient Sky Detection Algorithm from Fisheye Image Based on Region Classification and Segment Analysis
}

\author{
${ }^{1}$ Houssam Bourr, ${ }^{1}$ Youssef El Merabet, ${ }^{1}$ Rochdi Messoussi, ${ }^{1}$ lbtissam Benmiloud, ${ }^{2}$ Yassine Ruichek, \\ ${ }^{1}$ LASTID Laboratory, Department of Physics, \\ Faculty of Sciences, Ibn Tofail University \\ Kenitra, Morocco \\ ${ }^{2}$ Le2i FRE2005, CNRS, Arts et Metiers, Univ. Bourgogne Franche-Comte \\ Belfort, France \\ bourr.houssam@gmail.com; y.el-merabet@univ-ibntofail.ac.ma; \\ yassine.ruichek@utbm.fr; messousi@gmail.com
}

\begin{abstract}
In this paper, an efficient approach for automatic and accurate sky region detection from fisheye images is proposed. The proposed approach starts by segmenting the acquired image into regions using Statistical Region Merging method. After that, the segmented regions are characterized using local RGB color descriptor using image quantization. The next step consists of classifying the characterized regions into sky and non-sky regions by using maximal similarity based region classification through Hellinger kernelbased distance. In order to improve the obtained region classification results, a segment analysis based technique using Line Segment Detector is proposed. Experimental results prove the robustness and performance of the proposed procedure.
\end{abstract}

Region classification, RGB color descriptor, Segment analysis, LSD, Hellinger kernel-based distance.

\section{Introduction}

Sky region detection is necessary for many applications and it is one of the most significant subject matter commonly seen in outdoorimages. In the application of autonomous ground robot navigation, a robot should understand its surrounding environment by visual sensors; A precise sky detection can improve efficiency of road detection for independent ground robot. Sky detection can improve accuracy of GNSS based localization $[3,5,9]$, can be used for scene classification, and is often employed to achieve more effective content-based image retrieval [6]. Most existing sky region detection algorithms are mainly based on color priors $[1,2,11]$. In the method proposed in [1], both image simplification and classification are employed. First, the acquired image is simplified using a geodesic reconstruction [10] with an optimal contrast parameter. Second, the two classes (sky and not-sky) are classified from the simplified image. For that, a set of supervised (Bayes, KNN and SVM) and unsupervised (Fuzzy C-means, KMlocal,Statistical Region Merging and Fisher) clustering algorithms are compared with the purpose todefine the best classifier in terms of good classification rate. The method proposed by El merabet et al. [2] is constituted of four major steps: (I) A simplification step that consists in simplifying the image with an appropriate 
couple of colorimetric invariant and exponential transform. (II) The second step segments the simplified image in different regions of interest using Statistical Region Merging segmentation method. (III) The third step, the segmented regions are represented with a number of local color image region descriptors. (IV) The fourth step applies a supervised MSRC (Maximal Similarity Based Region Classification) method by using Bhattacharyya coefficient-based distance to classify the characterized regions into sky and non-sky regions.

Unfortunately, this color information based sky region detection approaches are often accompanied with false positives especially for some challenging cases like presence of buildings with the same color of sky (generally blue, white), or windows with mirrors or even mirrored buildings that reflect the color of sky. The purpose of this research is the development of an algorithm for sky detection from fisheye images with more accuracy, which should effectively deal with the above mentioned drawbacks. The method we propose is composed of two main phases: 1) an image-content classification method is used in order to classify the regions of the image into sky and non-sky and 2) an LSD [13] (Line Segment Detector) based segment analysis technique is performed in order to refine the obtained classification results.

The paper is organized as follows: Section 2 presents the image-content classification method employed in this research. Section 3 presents the LSD based segment analysis technique introduced to refine the classification results obtained with the image-content classification method presented in Section 2. Experimental results are shown in Section 4. Conclusions and future works are derived in Section 5.

\section{Image-Content Classification Method}

In this work, in order to obtain the preliminary classification results of the acquired fisheye images into sky and non-sky regions, we use the method introduced in [4]. This method is composed of several steps (cf. Figure 1): 1/ image simplification, 2/ image segmentation, 3/ region features extraction and 4/ region classification. these steps will be detailed in next sections.

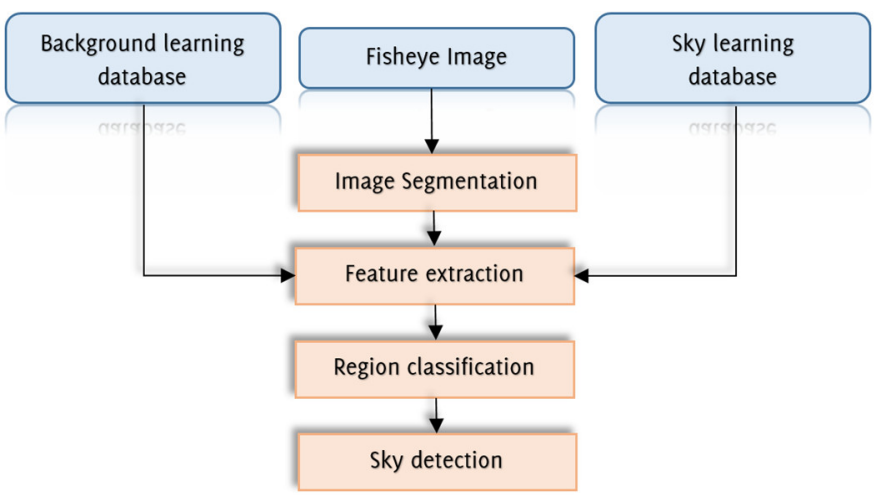

Figure 1: Flowchart of the image-content classification method.

\subsection{Initial segmentation using Statistical Region Merging}

As pointed out previously, the first step of the framework introduced in previous work of the authors [4] consists in partitioning acquired images into homogeneous regions with the same properties. Obviously, poor segmentation results could potentially impact the system reliability, i.e., the quality of classification results provided by the proposed procedure is strongly dependent on the segmentation results. In this work, in order to obtain a preliminary fisheye image segmentation, we have, like [4], used SRM (Statistical Region Merging) algorithm [8] that seems to be more adapted when considering the objectives of our 
Houssam Bourr, Youssef El Merabet, Rochdi Messoussi, Ibtissam Benmiloud, Yassine Ruichek, An Efficient Sky Detection Algorithm from Fisheye Image Based on Region Classification and Segment Analysis. Transactions on Machine Learning and Artificial Intelligence, Vol 5 No 4 August (2017); p: 714-724

application. SRM algorithm presents several advantages: 1 / it allows defining a hierarchy of partitions; 2 / it runs in linear-time by using bucket sorting algorithm while traversing the region adjacency graph (RAG); 3/ it dispenses dynamical maintenance of RAG; and 4/ it does not only consider spectral, shape and scale information, but also has the ability to cope with significant noise corruption and handle occlusions. Figure 2 illustrates an example of segmentation result via SRM algorithm.

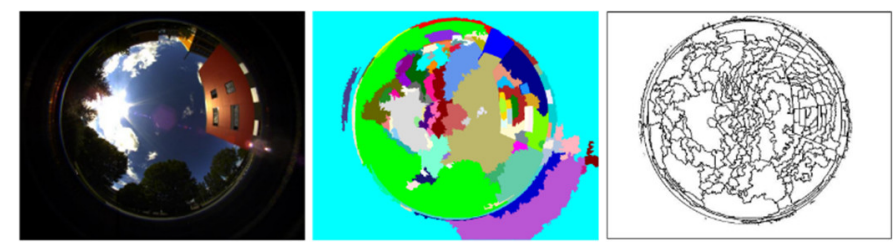

Figure 2: Segmentation result using Statistical Region Merging (SRM) method. From left to right: initial image, its SRM segmentation result and corresponding region map.

\subsection{Region representation}

The main goal of this research consists in identifying the regions corresponding to sky in fisheye images. For this purpose, we need first to characterize the segmented regions, obtained by using SRM algorithm, with a suitable descriptor. CEe extraction of this local image region descriptor, used as input to the region classification algorithm, permits to provide a global region representation with high level description than raw image pixels allowing thus, to discriminate robustly between the different regions in the treated images. In the present study, we have used RGB color histogram which is implemented as follows: each RGB color channel is first uniformly quantized into I levels, after that, the color histogram of each segmented region is produced in the feature space of $z=|\times| \times \mid$ bins. Let I be an image containing $N$ pixels quantized in $z=16 \times 16 \times 16=4096$ color bins, the $R G B$ color histogram of a segmented region $\mathcal{R}$ is represented as.

$$
h^{R G B}(\mathcal{R})=\left[h_{\mathcal{R}}^{1}, h_{\mathcal{R}}^{2}, \ldots, h_{\mathcal{R}}^{Z}\right]
$$

Where

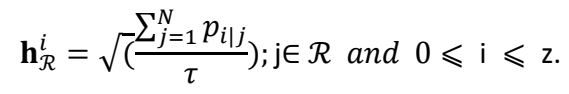

$\mathbf{h}_{\mathcal{R}}^{i}$ is the $i$ th normalized histogram bin and $\tau=\operatorname{card}(\mathcal{R})$ is the number of pixels in the region $\mathcal{R}$. $p_{i \mid j}$ is the conditional probability of the selected $j$ th pixel belonging to the ith color bin. It is expressed as follows:

$$
p_{i \mid j}=\left\{\begin{array}{c}
1, \text { if the } j \text { th pixel is quantized into the ith color bin } \\
0, \text { otherwise }
\end{array}\right.
$$

\subsection{Region classification}

In this stage of our method, we dispose of segmented regions $\mathcal{M}_{\text {SRM }}$ obtained via the SRM algorithm and which are characterized using the RGB color histogram as local image region descriptor. the challenge herein is to classify these characterized regions into sky and non-sky regions. To this end, we use the supervised region classification method introduced in [4]. Since this algorithm is a supervised procedure, we have first created two learning databases $\mathcal{B}_{\text {obj }}$ and $\mathcal{B}_{\text {back }}$ that are constructed respectively with $\mathrm{m}$ distinctive textures of sky regions and $n$ distinctive textures of non-sky regions such as building, road, tree, etc. Figure 3 illustrates the two constructed learning databases $\mathcal{B}_{\text {obj }}$ and $\mathcal{B}_{\text {back }}$ used in this work. Algorithm 
1 consists first in calculating the similarity between the characterized regions $\left(\mathcal{R} \in \mathcal{M}_{\mathrm{SRM}}\right)$ and those of the two learning databases $\mathcal{B}_{\text {obj }}$ and $\mathcal{B}_{\text {back }}$ through Hellinger kernel-based distance which represents the cosine of angle between the unit vectors representing the two regions to be compared:

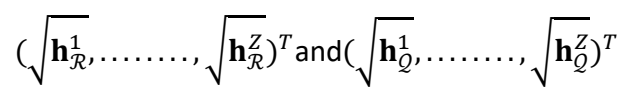

where $\mathbf{h}_{\mathcal{R}}^{i}$ is the normalized histogram of the region $\mathcal{R}$, the superscript $i$ represents its $i$ th element. $z=|x| x \mid$ $=4096$ represents the feature space. Note that the higher the Hellinger distance $\mathrm{e}(\mathcal{R}, \mathcal{Q}$ ) (cf. Eq. 4) between regions $\mathcal{R}$ and $\mathcal{Q}$ is, the higher the similarity between them is. That is to say that the angle between the two histogram vectors is very small involving that their histograms are very similar.

$$
\varrho(\mathcal{R}, Q)=\sum_{i=1}^{z} \sqrt{\mathbf{h}_{\mathcal{R}}^{i} \cdot \mathbf{h}_{Q}^{i}}
$$

After that, the algorithm assigns an unknown region $\mathcal{R}$ to a class $\mathcal{C}_{n}$, if the average of the $K$ first high similarity measures calculated between the region $\mathcal{R}$ and the regions of the learning database corresponding to the class $\mathcal{C}_{n}$ is maximal, i.e,

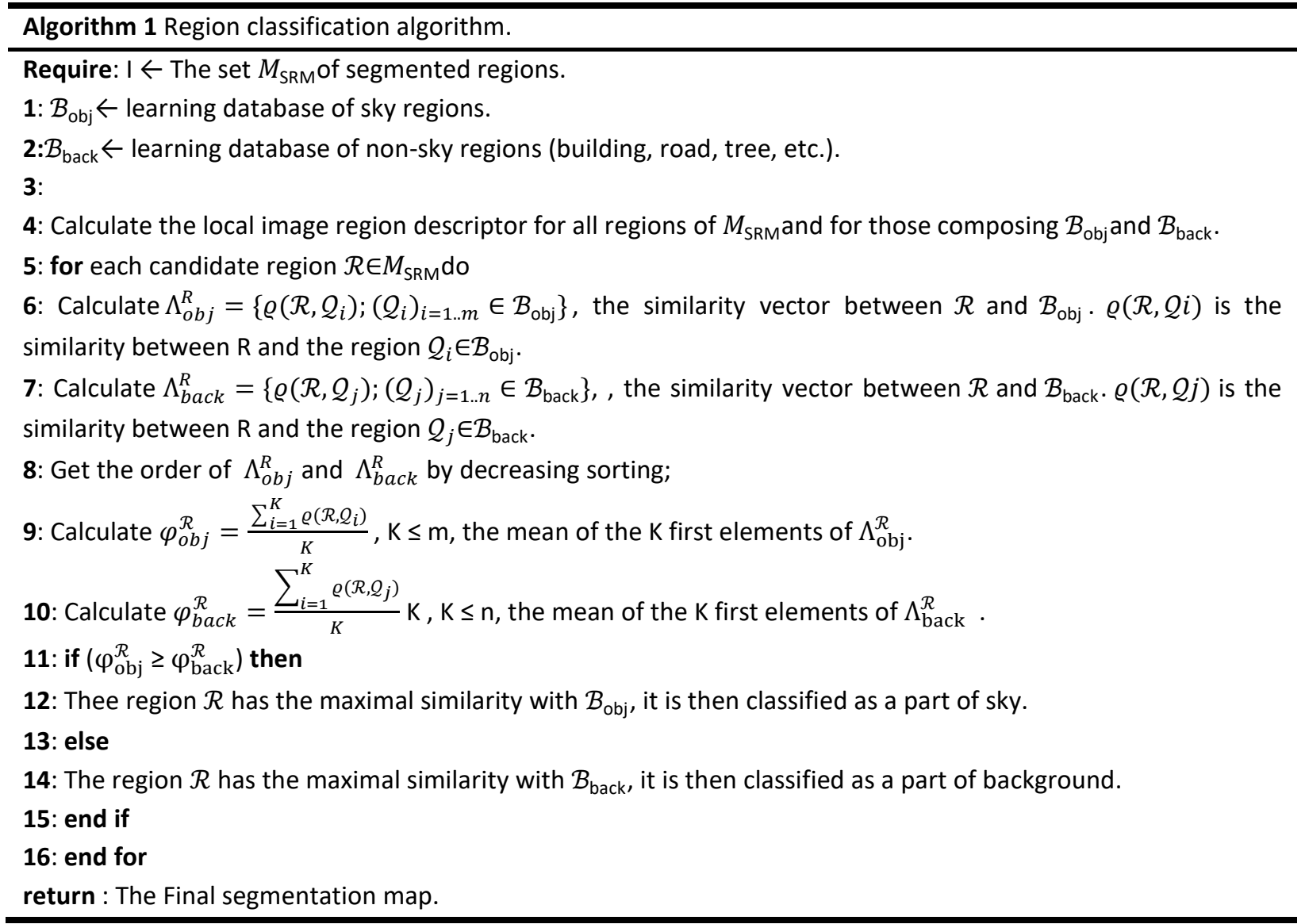

$$
C^{*}(\mathcal{R})=\underset{C_{n} \in C}{\operatorname{argmax}} \frac{1}{K} \sum_{i=1}^{K} \varrho\left(\mathcal{R}, Q_{i}\right), Q_{i} \in B_{n}, n=1,2
$$


Houssam Bourr, Youssef El Merabet, Rochdi Messoussi, Ibtissam Benmiloud, Yassine Ruichek, An Efficient Sky Detection Algorithm from Fisheye Image Based on Region Classification and Segment Analysis. Transactions on Machine Learning and Artificial Intelligence, Vol 5 No 4 August (2017); p: 714-724

where $\mathcal{B}_{1}$ and $\mathcal{B}_{2}$ are the learning databases corresponding to the classes $\mathcal{C}_{1}$ and $\mathcal{C}_{2}, \mathcal{R}$ is a query, and $\varrho$ is the similarity measure.
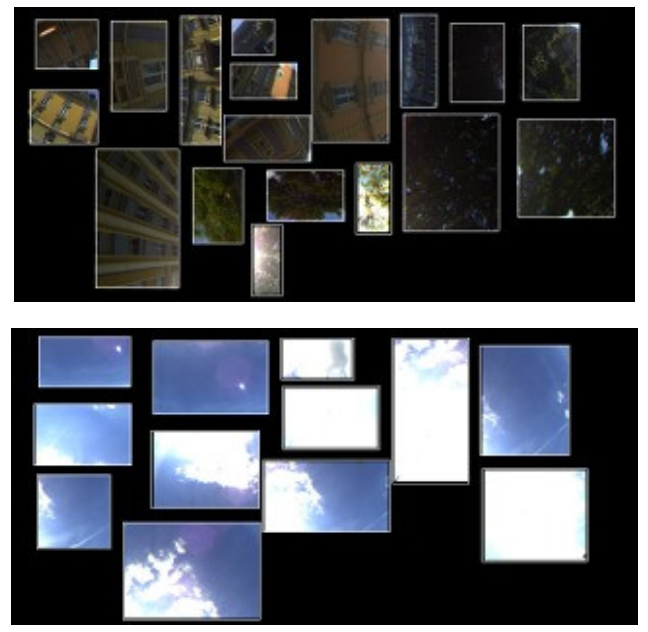

Figure 3: Example of learning databases used in this work. From top to bottom: learning database $\mathcal{B}_{\text {back }}$ of background (vegetation, building, etc) and learning database $\mathcal{B}_{\text {obj }}$ of sky.

\section{Segment Analysis Technique}

The region classification method introduced previously allows achieving good classification results. Unfortunately, in some challenging cases like the presence of buildings with the same color as sky region (generally blue and white colors), windows with mirrors or even mirrored buildings that reflect the color of sky region, the method performs worse. Indeed, the method is failing to distinguish between building and sky regions with the same color using only color information in the region descriptor, it is then accompanied with false positives. Figure 4 illustrates some false detections generated by Algorithm 1 described above. To deal with this shortcoming and therefore improve sky region detection results, we propose to couple Algorithm 1 with a segment analysis procedure. This strategy is based on a detection of segments on which we establish two hypotheses. The first one supposes that the confusion between the vegetation and sky regions is highly impossible via Algorithm 1. the second hypothesis postulates that buildings, referring to their constitution, contain a significant number of segments. The final sky detection strategy we have developed consists of four steps described below:

(1) Detection of segments in the image.

(2) Creation of an optimized mesh.

(3) Conversion into a density map.

Region classification via the whole proposed approach
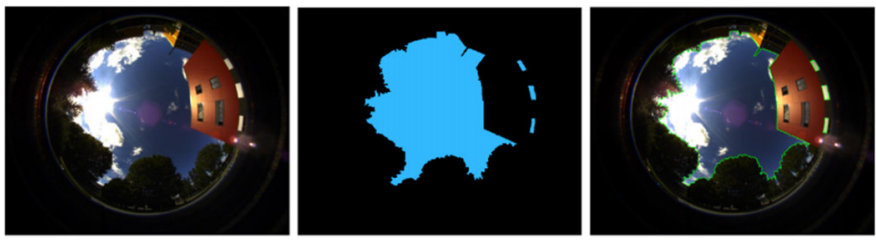

Figure 4: False detections generated by Algorithm 1. 


\subsection{Segments detection}

This step constitutes the cornerstone of the proposed alternative to overcome the shortcoming of Algorithm 1 and thus will improve the system reliability in terms of classification of the content of fisheye images into two classes (sky, not sky). Many methods of segments detection exist in the literature like Hough transform, Dseg, LSD [13], etc., however many of them make their parameters optimized for a targeted application. According to one of our application constraints, related to the optical sensor used: a color camera with a fisheye lens providing a field of view of more than 180 degrees with significant distortions, we have opted to use the algorithm LSD which others the best results. The LSD (Line Segment Detector) method is a segment detection technique that focuses on the orientation of the gradient and a strict selection of the segments detected. Figure 5 illustrates an example of segments detection on both grayscale and geodisk reconstruction by dilation (GRD) images. Note that the detection results obtained with the LSD algorithm in the framework of our application are promising since we have a large number of segments detected in the building regions and little in the other regions (vegetation, trees, etc.) of the image.
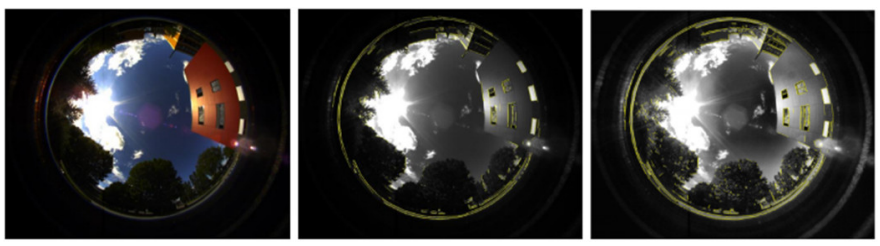

Figure 5: Detection of segments with LSD. From left to right: the original image, detected segments on grayscale and on GRD images.

\subsection{Creation of an optimized mesh}

As pointed out in the previous step, we have obtained a high density of segments detected in the buildings but also on their contours. The next step consists in connecting different segments to create shapes with closed contours which will be labeled to create a map of regions coinciding with building areas. Fe objective herein is to refine the classification results obtained by Algorithm 1, i.e., reclassify building regions which are classified as true positive (sky region). For that, the creation of the mesh concerns only the detected segments composed of non-sky pixels. That being said, the segments having one of their extremities present in sky area are removed to avoid unnecessary connections of segments in sky regions. During the segment connection step, only the segments where the corresponding extremities are very close to each other in a Euclidean distance sense are connected by a new segment. The tests we have carried out conclude that a value of 20 pixels (distance between extremities of two segments candidates to connection) gives good results. Figure 6 illustrates an example of mesh obtained from segments detected in an image. As can be seen from this figure, the mesh result shows a large concentration of small-sized regions in the building areas.

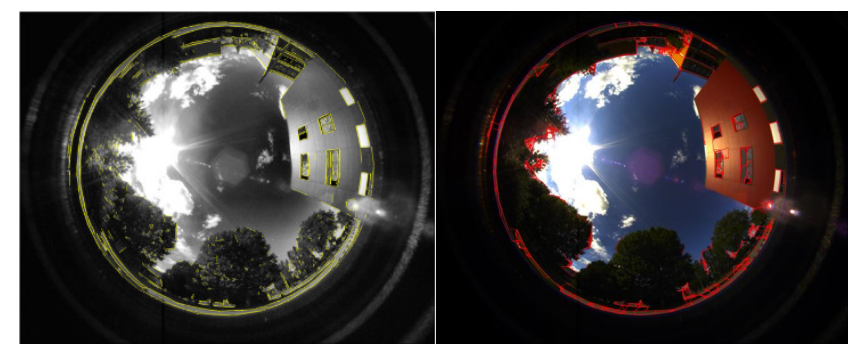

Figure 6: Illustration of segment detection (left), and creation of a mesh (right). 


\subsection{Conversion into a density map}

The so called density map is obtained by filling all the holes in the mesh obtained in the previous step. Figure 7 illustrates the density map corresponding to the mesh of Figure 6 . These results, which confirm the hypotheses previously mentioned, are therefore very satisfactory.

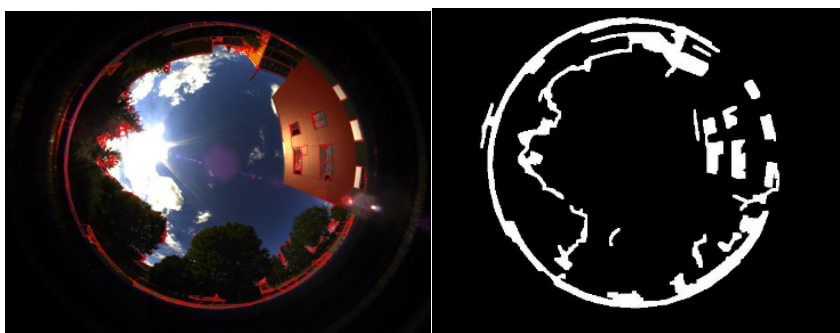

Figure 7: Illustration of the creation of a mesh (left), density map (right).

\subsection{Region classification via the whole proposed approach}

It is a question herein to define the class to which each region $\mathcal{R}$ belongs among the two possible classes: sky and non-sky, from the region map and the density map previously calculated (cf. Figure 8). The use of the density map allows each region to be assigned a density percentage, i.e., the ratio between the number of pixels belonging to the density map within the candidate region $\mathcal{R}$ and the total number of pixels of the same region. In order to optimize and strengthen decision-making on classes to which image regions belong, we start by considering that each region $\mathcal{R}$, initially classified as sky region via Algorithm 1 , whose density percentage is less than $90 \%$ of the image, will be automatically classified as sky region. In contrary, if this percentage is greater than $90 \%$, whatever the classification result of Algorithm 1 (sky or non-sky), the region will be classified as non-sky region. Let $\mathcal{R} \in$ region map and $P_{\mathrm{R}}$ is the density percentage. The class to which the region $\mathcal{R}$ belongs is determined using Eq 6 . Algorithm 2 illustrates the steps of the whole proposed approach.

$$
R=\left\{\begin{array}{ccc}
\text { sky } & \text { if } & P_{R}<90 \% \text { et } \Omega(R)=R_{\text {sky }} \\
\text { non }- \text { Sky } & \text { if } & P_{R}>=90 \%
\end{array}\right.
$$

where $\Omega$ is the set of obtained sky/non-sky regions via Algorithm 1.
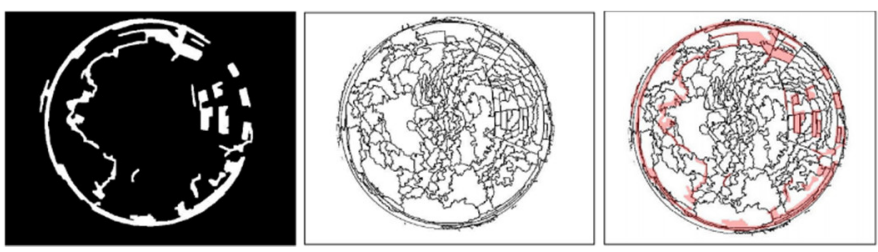

Figure 8: Merge of density and region (cf. Figure 2) maps. From left to right: density map, region map and the obtained merging result.

\footnotetext{
Algorithm 2 Proposed approach

Require: $I \leftarrow$ input image.

$M_{\text {SRM }} \leftarrow$ SRM Area Map.

$\Omega \leftarrow$ obtained sky/non-sky regions via Algorithm 1 .

1: $I_{\mathrm{GRD}} \leftarrow$ Apply the GRD contrast to the gray level (I).
} 


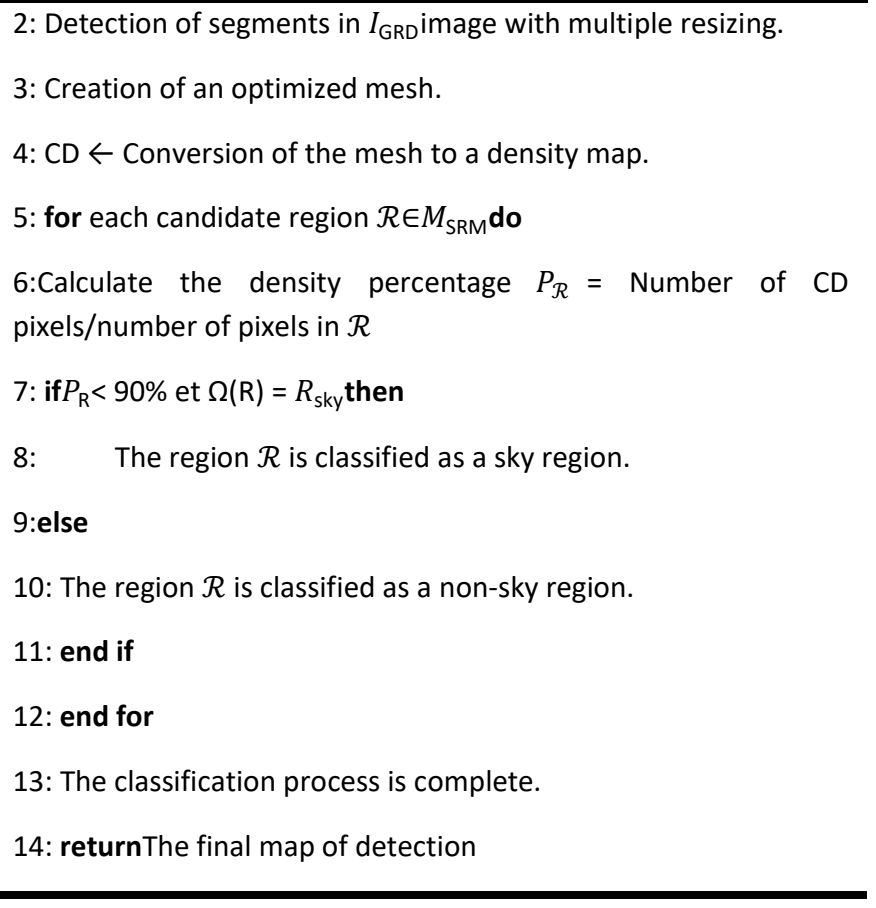

\section{Experimental Results}

In this section, we are interesting in assessing the ability of the proposed approach to deal with the detection of sky zones from fisheye images. We begin by a qualitative evaluation of the proposed method using representative test images. Figure 9 illustrates an example of visual comparison of the region classification results. From top to bottom: classified image into two classes (sky and non-sky) obtained by Algorithm 1 and classification result obtained by the proposed approach. In Figure 9, the sky boundaries are drawn with green color and superimposed upon the initial images. Basing on visual evaluation of these results, one can see clearly that, due to radiometric similarity between some pixels belonging to building and sky regions, several non-sky parts are classified as true positive (sky region). In contrary, from the second row of Figure 9, it is clear that the proposed approach demonstrates excellent accuracy in terms of sky boundary extraction. This means that the majority of the sky zones present in the images are detected with good boundary delineation. Indeed, our method shows reliable results across complex environment composed of sky, building, road areas, vegetation, etc.

As for quantitative evaluation, we use measures widely employed in evaluating effectiveness. They constitute a useful and accepted tool in object recognition field [12]. The 150 fisheye images of the tested dataset were first manually delineated. then, they are used as a ground truth image segmentation to assess the accuracy of automated sky regions extraction. The obtained results and reference ones are compared pixel-by-pixel. Each pixel in the images is categorized as one of the four possible outcomes:

(1) True positive (TP): Both manual and automated methods label the pixel belonging to sky.

(2) True negative (TN): Both manual and automated methods label the pixel belonging to background.

(3) False positive (FP): The automated method incorrectly labels the pixel as belonging to sky. 

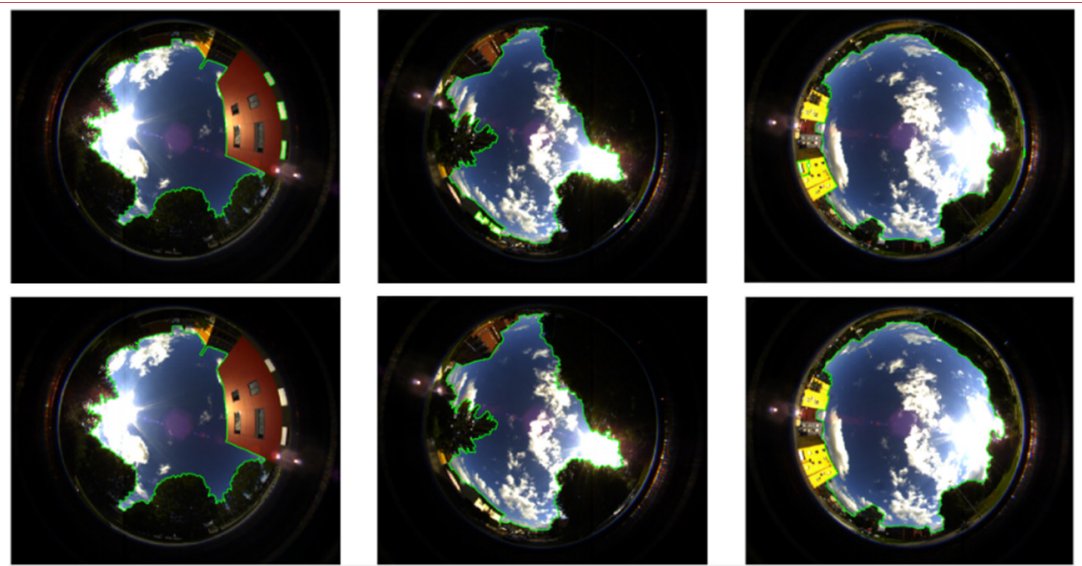

Figure 9: Visual comparison of region classification results. From top to bottom: classified image into two classes (sky and non-sky) obtained by Algorithm 1 and classification result obtained by the proposed approach.

Table 1: Evaluation results using Algorithm 1 and the proposed approach (Algorithm 2)

\begin{tabular}{cccccc}
\hline Method & Recall (\%) & Precision (\%) & F-measure (\%) & Accuracy (\%) & MMC (\%) \\
\hline Algorithm 1 & 98.66 & 96.36 & 97.48 & 99.00 & 0.97 \\
Proposed approach (Algorithm & 98.90 & 97.48 & 98.18 & 99.10 & 0.98 \\
2) & & & & \\
\hline
\end{tabular}

(4) False negative (FN): The automated method does not correctly label the pixel truly belonging to sky.

To examine detection performance, the number of pixels that fall into each of the four categories TP, TN, FP, FN are determined, and the following measures are computed:

$$
\begin{gathered}
\text { Recall }=\frac{T P}{P} \times 100 \\
\text { Precision }=\frac{T P}{T P+F P} \times 100 \\
F 1=2 \times \frac{\text { Precision } \times \text { Recall }}{T P+F P} \\
\text { Accuracy }=\frac{T P+T N}{T P+F P} \times 100 \\
\text { Recall }=\frac{(\mathrm{TP} \times \mathrm{TN})-(\mathrm{FP} \times \mathrm{FN})}{\sqrt{(\mathrm{TP}+\mathrm{FP}) \times(\mathrm{TP}+\mathrm{FN}) \times(\mathrm{TN}+\mathrm{FP}) \times(\mathrm{T} \mathrm{N}+\mathrm{FN})}}
\end{gathered}
$$

Table 1 summarizes the average (on the dataset) of the performance indicators recall, precision, F1 measure, Accuracy and MCC according to Algorithm 1 used alone on the one hand and with the proposed segment analysis technique. It can be clearly seen that the proposed method maximizes the performance indicators compared to Algorithm 1 used alone. Indeed, the proposed approach demonstrates its effectiveness as it shows superior performances, which confirms hence the conclusion given from the analysis of Figure 9. 


\section{Conclusion}

In this paper, an automatic sky region detection method is proposed. The procedure we proposed includes a region based classification method and a segment analysis based technique. The first method is used in order to classify segmented regions via Statistical Region Merging, characterized by RGB color descriptor, into two classes (sky and non-sky regions). CEe second method is introduced in order to refine the classification results, especially the regions belonging to building area which were mistakenly classified by the first method as true positive (sky region). As highlighted by the obtained results, the proposed approach is therefore able to distinguish perfectly the sky/non-sky areas in a fisheye image. For future works, we plan to improve the effectiveness of the proposed approach by proposing new color descriptors and evaluate the impact of other segment detection methods on the quality of the classification results.

\section{REFERENCES}

[1] Dhouha Attia. 2014. Segmentation d'images par combinaison adaptative couleur-texture et classification de pixels. : Applications la caractérisation de l'environnement de réception de signaux GNSS. PhD thesis, Université de ' technologie Belfort-Montbéliard(Jun 2014).

[2] Y El merabet, Y Ruichek, S Ghaffarian, Z Samir, T Boujiha, R Touahni, and R. Messoussi. 2016. Horizon Line Detection from Fisheye Images Using Color Local Image Region Descriptors and Bhattacharyya CoefficientBased Distance. In International Conference on Advanced Concepts for Intelligent Vision Systems. Springer International Publishing. 58-70.

[3] Juliette Marais et Cyril Meurie. 2013. Quantify et improve GNSS quality of service in land transportation by using image processing. First CNES-ONERA Workshop on Earth-Space Propagation, hal-00912681 (December 2013).

[4] A. El Idrissi, Y. El Merabet, Y. Ruichek, R. Touahni, A. Sbihi, C. Meurie, and A. Moussa. 2015. A MultipleObjects Recognition Method Based on Region Similarity Measures: Application to Roof Extraction from Orthophotoplans. (IJACSA) International Journal of Advanced Computer Science and Applications, Vol. 6, No. 11 (Novembre 2015).

[5] J. Marais, C. Meurie, S. Ambellouis, and Y. Ruichek. 2013. Projet CAPLOC. Combinaison de l'analyse d'images et la connaissance de la propagation des signaux pour la Localisation. (Agust 2013).

[6] L. Matthies, M. Maimone, A. Johnson, C. Yang, R. Willson, C. Villalpando, S. Goldberg, A. Huertas, A. Stein, and A. Angelova. 2007. Computer vision on mars. Int. J. Comput. Vis. 75, 1 (2007), 67-92.

[7] J. Ning, L. Zhang, D. Zhang, and C. Wu. 2009. Interactive image segmentation by maximal similarity based region merging. (Mars 2009).

[8] R. Nock and F Nielsen. 2004. Statistical region merging. IEEE Trans. Pattern Anal. Machine Intelligence 26, 11 (2004), 1452-1458.

[9] S. Ramalingam, S. Bouaziz, P. Sturm, and M. Brand. 2010. SKYLINE2GPS: Localization in Urban Canyons Using Omni-Skylines. IEEE International Conference on Intelligent Robots and Systems (2010), 3816-3823. 
Houssam Bourr, Youssef El Merabet, Rochdi Messoussi, Ibtissam Benmiloud, Yassine Ruichek, An Efficient Sky Detection Algorithm from Fisheye Image Based on Region Classification and Segment Analysis. Transactions on Machine Learning and Artificial Intelligence, Vol 5 No 4 August (2017); p: 714-724

[10] Z. B Sbeh, L. D Cohen, G Mimoun, and G Coscas. 2001. A New Approach of Geodesic Reconstruction for Drusen Segmentation in Eye Fundus Images. IEEE Transactions on medical imaging 20, 12 (Decembre 2001), 1321-1333.

[11] Y. Shang, G. Li, Z. Luan, X. Zhou, and G Guo. 2016. Sky detection by effective context inference. Neurocomputing 208 (2016), 238-248.

[12] J. A Shufelt. 1999. Performance evaluation and analysis of monocular building extraction from aerial imagery. IEEE Trans. Pattern Anal. Machine Intell 21 (1999), 311-326.

[13] R. G Von Gioi, J Jakubowicz, J. M Morel, and G Randall. 2012. LSD: a line segment detector. Image Processing On Line 2 (Mars 2012), 35-55. 